\title{
A Hybrid, Multi-Agent Model of Foraging Bottlenose Dolphins *
}

\author{
Musad Haque * Amir Rahmani * Magnus Egerstedt * \\ * Georgia Institute of Technology, Atlanta, GA 30332 USA \\ (e-mail: \{musad.haque, amir.rahmani, magnus\}@ece.gatech.edu)
}

\begin{abstract}
Social behavior of animals can offer solution models for missions involving a large number of heterogeneous vehicles, such as light combat ships, unmanned aerial vehicles, and unmanned underwater vehicles. We draw inspiration from the foraging techniques of bottlenose dolphins to address the problem of heterogeneous multi-agent herding. We produce a hybrid automaton model of the entire foraging method - search, detect, and capture - where agents are modeled as first-order systems in which interactions are defined through spatial proximity. Finally, simulations are provided to illustrate that our model is expressive enough to capture this complex biological phenomenon.
\end{abstract}

Keywords: Biologically-Inspired Methods, Hybrid Systems, Decentralized Control, Multi-Agent Herding, Networked Control Systems

\section{INTRODUCTION}

The foraging task is known to be a canonical testbed for cooperative robotics (Cao et al. (1997), Østergaard et al. (2001)). The problem of herding when a group of foraging robots confine the movement of another group of robots (active rather than passive objects) and move them to a pre-specified area has been the focus of much attention recently (Ferrari-Trecate $e t$ al. (2006)). We intend to address the problem within the framework of a heterogeneous system by drawing inspiration from foraging bottlenose dolphins, Tursiops truncatus. Our goal is to model this inherently hybrid biologically-complex foraging behavior in the context of multi-agent coordination. In Particular, we will produce a hybrid model that is rich enough to capture this complex biological phenomenon, yet lends itself to further analysis.

The bottlenose dolphins employ two very interesting group foraging techniques - the "wall method" and the "horizontal carousel" method - that testify to their complex social behavior. In both methods, a herd of dolphins will cooperate amongst themselves to manipulate the movement of their prey, which in this scenario, is a school of fish. In the wall method, the dolphins drive the fish towards a wall, which could be either the shore or other dolphins, and feed on the fish that bounce off the wall. In the horizontal carousel technique, the dolphins first encircle the fish and then slowly tighten this encirclement into smaller and smaller circles, consequently, constricting the movement of the fish into smaller and smaller bubbles. At one point, when the circle is small enough, the dolphins will start to feed by charging through the fish, either one at a time, or together at once. We would like to create a model of this complex biological phenomenon in the context of heterogeneous multiagent herding, where, a group of agents replicating foraging dolphin behavior will herd a group of agents replicating fish behavior.

\footnotetext{
ऋ The work by Musad Haque, Amir Rahmani, and Magnus Egerstedt was supported in part by the Office of Naval Research through MURI-HUNT.
}

Labella et al. (2004) presented a foraging strategy inspired by ant colonies. Ferrari-Trecate et al. (2006) use a stop/go policy for the herders by ensuring that the group of herded are always confined in the convex polytope of the herding agents. They achieve herding behavior by considering cooperation between herders and the herded robots whereas in our approach we attain confinement of non-cooperative robots.

Using tools from decentralized networked controls and hybrid systems, our goal is to produce a herding model that is rich enough to mimic the complex biological phenomena of the foraging bottlenose dolphins. Both classes of agents (dolphins and fish) are modeled as first-order networks with unicycle dynamics, where decisions by these autonomous agents are made solely based on local interactions with other agents.

\section{BOTTLENOSE DOLPHINS}

Bottlenose dolphins are very intelligent animals that also exhibit a wide range of complex social behaviors (see Pryor and Norris (1998), Mann et al. (2000) for further details). They live in fission-fusion societies (see Couzin (2006)) where the primary group breaks up into smaller groups to explore or forage and later rejoins the group to share resources (Mann et al. (2000)). These groups may have leaders but, they are in turn governed by an overall social hierarchy, where the largest dolphin is usually the most dominant (Pryor and Norris (1998)). Bottlenose dolphins also display cooperative behavior while defending other dolphins, during foraging and capturing prey, and while searching for mates (Schusterman (1986)).

During foraging, dolphins move in specific group formations, which are selected based on the threat level of the environment (Pryor and Norris (1998)). The distribution of killer whales and tiger shark (which are dolphin predators), fishing nets, and boating activities constitute of threats (Heithaus and Dill (2002)). The relative positions of the dolphins in each group are shown in Figure 1. These formations are adaptive since when the dolphins are near shore, where the threat level is high, they tend to stick together and form the "tight" formation, but 
when foraging further away from the shore, they spread out and switch to either "front" or "double front" formation. When dolphins finally locate their prey, they have at their disposal, a couple of very interesting trapping methods. The two methods, wall and horizontal carousel, are explained in detail in the following sections. The success of both methods depend on the dolphins' ability to constrict the "maneuverability of the prey" (Pryor and Norris (1998)).

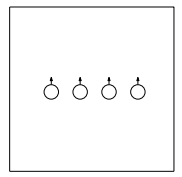

(a) Front

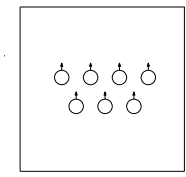

(b)

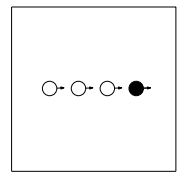

(c) Line

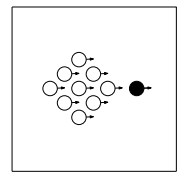

(d) Tight-group
Fig. 1. Types of bottlenose dolphin formations used during foraging. Some formations require a leader (shown in black) and the arrows denote headings of the dolphins.

\subsection{Wall Method}

The wall method is a foraging technique that is used quite often by the bottlenose dolphins. Pryor and Norris (1998) provide a detailed description of this prey-capturing method; in short, the dolphins "drive" the fish towards a barrier and capture them from the foam of returning water as shown in Figure 2. There are four variations of the wall method: 1) "fish in front," where a group of dolphins use the shore as a barrier; 2) "dolphin group as wall," where a second group of dolphins will act as the wall for the original group that drives the fish; 3) "two columns," where two groups of dolphins form parallel columns, with the school of fish trapped in between, and guide their prey to shore to perform "fish in front" once near the shore; and 4) "two frontal attack," where two groups of dolphins drive the fish towards each other.

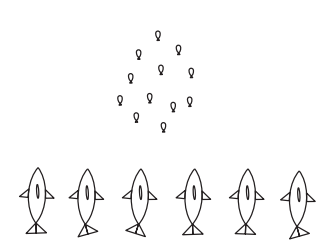

Fig. 2. Bottlenose Dolphins in a front formation driving fish against the shore.

\subsection{Horizontal carousel}

In the horizontal carousel, when the dolphins find a sizable amount of fish, they create a large circle to trap the fish inside it. The dolphins then start to tighten the encirclement, by forming smaller and smaller circles, to constrict the movement of their prey (see Mann et al. (2000)) as shown in Figure 3. At one point, the encirclements become small enough for the dolphins to dive into the school and feed on their prey. This carousel-like movement by the dolphins is initiated by either "curving" in from one side of the fish or by simultaneously surrounding them from both sides (Pryor and Norris (1998)). The eventual charge into the school of fish by the dolphins is done in two different ways - in the "vertical carousel" the dolphins dive underneath the school, one by one, in an orderly way and feed on their way up back, and in the "kettle method," the dolphins try to confuse the fish by diving into the school, all at once, from different directions. Since we are only concerned with foraging, for more information on these feeding techniques, see Pryor and Norris (1998).

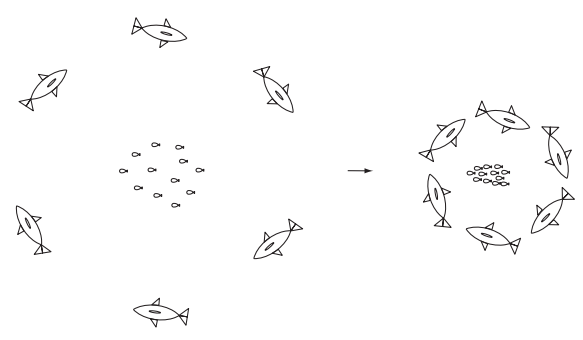

Fig. 3. Bottlenose dolphins tightening the encirclements around a school of fish.

\section{MULTI-AGENT HERDING}

The foraging process of bottlenose dolphins is classified into three phases - search, detect and capture (Pryor and Norris (1998)). In the search phase, dolphins look for prey by either sending out scouts ( 2 dolphins), by forming groups $(2-6$ dolphins), or by foraging together as a herd as shown in Figure 4. In the detect phase, the dolphin that finds prey notifies the position of the prey to the rest of the herd, and subsequently, in the final phase, a group of dolphins will converge to that location and employ a capturing method (some variation of the wall method or the carousel method) to trap and feed on their prey. Next, we discuss how a multi-agent system (with only locally-interacting agents and unicycle dynamics) can be designed to reproduce this complex biological behavior.

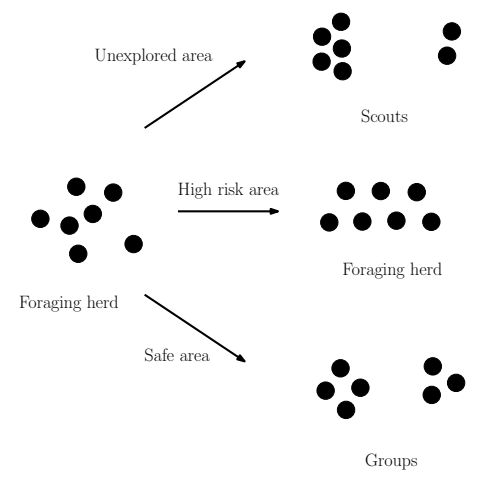

Fig. 4. Dolphins forage in three ways - using scouts, as groups, or together as a herd.

\subsection{Search Method Selection by Leader Agents}

As mentioned before, dolphins have a very well defined social hierarchy, where the role of the dominant dolphin goes to the largest male. Pryor and Norris (1998) explains the roles of this dolphin in detail, but to summarize, the most dominant dolphin in the herd plays two very important roles: 1) it determines the threat level of an environment and 2) it is the first to check out an unexplored area.

We need to first establish this notion of dominance for our multi-agent system. We assign each agent $i \in \mathcal{H}$ (we use $\mathcal{H}$ to denote "herd"), a dominance factor $d_{i} \in \mathbb{R}$. We assume that there exists an agent $l \in \mathcal{H}$ such that $d_{l}>d_{i}, \forall l \neq i \in \mathcal{H}$. With this formulation, we establish the presence of a "leader" agent within the entire set of agents. Since the herd can split up into groups, we also assume every group within the herd is 
also led by a dominant dolphin from within the group. Thus, there is a dominant dolphin that leads the entire herd, but when a task requires them to cluster into groups, each group is also led by a dominant dolphin from within the group. If there are $N_{G}$ groups $\left(N_{G}=1\right.$ when the entire herd forages together) that emerge from the herd, then we have $\mathcal{H}=\cup_{j=1}^{N_{G}} \mathcal{G}_{j}$ such that $\mathcal{G}_{j} \neq \varnothing$ and $\mathcal{G}_{j} \cap \mathcal{G}_{i}=\varnothing, \forall j \neq i \in\left\{1, \ldots, N_{G}\right\}$.

As mentioned earlier, we assume that each group also have a leader, i.e., there exists an agent $l_{j} \in \mathcal{G}_{j}$ such that $d_{l_{j}}>$ $d_{i} \forall l_{j} \neq i \in \mathcal{G}_{j}$. The set of leaders, $\mathcal{L}$, is given by $\mathcal{L}=\left\{l_{j} \mid l_{j} \in\right.$ $\left.\mathcal{G}_{j} \forall j \in\left\{1, \ldots, N_{G}\right\}\right\}$. Without loss of generality we assume the most dominant agent in the entire herd belongs to the first group and denote it as $l_{1}$.

Since the dominant dolphin is always monitoring the threat level of the environment, we define a function $p^{r}: \mathcal{L} \rightarrow \mathbb{R}$ to characterize the threat assessment ability of leader agents. Thus, $p^{r}(i)$ denotes the threat level of the environment assessed by agent $i \in \mathcal{L}$ (superscript " $\mathrm{r}$ " stands for risk). The critical threat levels $r_{\min }$ and $r_{\max }$ will be used to classify predator risk into different levels, where $0<r_{\min }<r_{\max } \in \mathbb{R}$. In our model, we allow group leaders to monitor the threat level, in addition to the leader agent $l_{1}$, to avoid the scenario where the agent $l_{1}$ declares an area to be safe while a group leader further away is being encountered by a predator.

If the dolphins are foraging in a previously unexplored area, then the dominant dolphin will form a group of "scouts" with other lesser-dominant dolphins in the herd to examine the area while searching for food, while the main herd in turn, follows these scouts from a safe distance. We characterize the familiarity of a foraging area through the function area $: l_{1} \rightarrow$ $\{0,1\}$ such that area $\left(l_{1}\right)=1$ denotes that the current foraging area has previously been used to forage, while area $\left(l_{1}\right)=0$ represents a previously unexplored foraging area. Notice that this assessment is done by the most dominant agent in the herd, $l_{1}$, and when area $\left(l_{1}\right)=0$, the dolphins will search for food using scouts; otherwise, the dolphins will either forage in groups or as a whole herd. At the start of the foraging process, a suitable search method is chosen by the leader agent $l_{1}$, depending on the risk of predation and the familiarity of the foraging area using the following criteria:

$$
\text { search }=\left\{\begin{array}{l}
\text { scouts if } \quad \text { area }\left(l_{1}\right)=0 \wedge p_{\text {max }}^{r}<r_{\max } \\
\text { group if } \quad \text { area }\left(l_{1}\right)=1 \wedge p_{\max }^{r}<r_{\min } \\
\text { herd if } \quad \text { area }\left(l_{1}\right)=1 \wedge r_{\min } \leq p_{\max }^{r}<r_{\max }
\end{array}\right.
$$

where $p_{\max }^{r}=\max _{i} p^{r}\left(l_{i}\right)$. Notice that if the threat level is higher than $r_{\max }$ before foraging begins, then the leader agent will prevent the dolphins from starting the process.

\subsection{Communication Topology}

Dolphins use their echolocation system to communicate and this constitutes of using sonar and making "rapid clicks" (see Cousteau and Diol (1975); Mann et al. (2000)). We model this limited range communication by a graph where dolphin agents form its vertices and an undirected edge set $(i, j) \in \mathcal{E}$ if $\left\|x_{i}(t)-x_{j}(t)\right\| \leq \Delta$, where $\Delta$ is the communication range. We further assume that group leaders always remain connected, i.e., $\left(l_{i}, l_{j}\right) \in \mathcal{E}, \forall l_{i}, l_{j} \in \mathcal{L}$. We define the neighborhood set, $\mathcal{N}_{i}$, of each agent $i$ as the collection of all agents in the $\Delta$ range of it.
We postulate the nearest-neighbor rule to model both the interdolphin and inter-fish interactions. In Schusterman (1986), it is observed that individual dolphins in a group move with a virtual bubble around them that other members do not enter. Moreover, small fish generally maintain a constant "inter-individual distance" as described in Hoare et al. (2004) and from a networked control point of view, this implies that each agent is aware of the position of its neighbors. As a result, the agents in our model will be able to calculate the relative displacements between itself and its neighbors.

\subsection{Detection}

When a dolphin encounters a school of fish, if there is enough prey available for a group feeding to take place, the rest of the herd will come and join this "advertising" dolphin (Pryor and Norris (1998)). This implies that the location of the prey is sent to the other dolphins and that dolphins are capable of measuring the biomass of the prey they encounter. We instill this measuring capability in our agents through the function $p^{f}: \mathcal{H} \rightarrow \mathbb{R}$. Thus, $p^{f}(i)$ represents agent $i$ 's estimate of the amount of prey it encounters (superscript " $f$ " stands for fish). If this estimate is greater than some threshold, $f^{\star} \in \mathbb{R}$, then the agent will "advertise" the location of the prey to the rest of the herd. In our formulation, the location being broadcasted is the centroid of the prey, $\rho$. The advertising aspect is recreated by the function $a d v: \mathcal{H} \rightarrow\{0,1\}$, defined as follows:

$$
a d v(i)= \begin{cases}1 & \text { if } p^{f}(i) \geq f^{\star} \\ 0 & \text { otherwise }\end{cases}
$$

Due to the limited communication range of our agents, $a d v(i)=$ 1 translates to agent $i$ locating prey, large enough for group feeding, and broadcasting this information to its one-hop neighbors. However, since we already assumed that our graph is connected during foraging, the leader agent, $l_{1}$, will receive this information as seen in Figure 5. In our framework, once, the leader agent receives the location of prey, it will initiate the capturing phase. In order to capture the fish, the leader must decide which capturing method to use and which dolphins to employ for that method. The next section will discuss how the leader makes these selections.

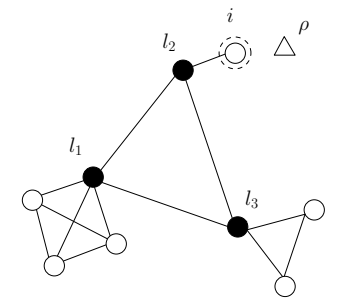

Fig. 5. The agents (circles) are searching in groups. The lines denote edges and leaders (shown in black) remain connected during foraging. The agent $(i)$ advertising the centroid of prey (triangle) has a ring around it.

\subsection{Capture Method Selection Through Voting}

As mentioned before, we have five possible methods to capture prey - four variations of the wall method and the carousel method. Let us label these methods for simplicity of notation: "fish in front" as method 1, "two frontal attacks" as method 2, "group as a wall" as method 3, "two columns" as method 4, and "carousel" as method 5. Observations presented in Pryor and Norris (1998) show that the number of dolphins participating in 
the different methods can often be divided into distinct ranges; for example, $5-15$ dolphins are usually found in the carousel method. Since agents in our model makes decisions based on local interactions, an agent will choose a method based solely on the number of neighbors it has when it receives the location of the prey.

Define voting profile of agent $i$ as $m(i) \in \mathbb{R}^{5}$; a column vector with 1 in the row corresponding to the voted method and zero everywhere else. Agent $i$ selects method $k$, i.e. $m_{k}(i)=1$, if $\left|\mathcal{N}_{i}\right| \in\left[N_{k}, N_{k+1}\right)$, where $N_{k} \in \mathbb{N}, N_{k+1}>N_{k} \forall k \in$ $\{1 \ldots 5\}$, and $|\cdot|$ denotes cardinality. Furthermore, we let $N_{1}=1$ and $N_{6}=|\mathcal{H}|$ to eliminate the possibility of a no vote.

We still need to establish coordination between agents to avoid a scenario where one group of dolphins is performing a wall method while another group is performing the carousel method to catch the same school of fish. We need a global behavior to emerge from these local opinions and the way we are going to select a suitable method for the entire herd is through a weighted poll conducted by the leader agent, where the weights will correspond to the dominance of each agent.

Let $\bar{m}_{k}$ denote the votes for method $k$ tallied by the leader agent, $l_{1}$. This is calculated as follows:

$$
\bar{m}_{k}=\sum_{i=1}^{N}\left(d_{i} \cdot m_{k}(i)\right) \forall k \in\{1 \ldots 5\}
$$

The leader will direct the herd to use method $k$ if $\bar{m}_{k}>$ $\bar{m}_{n} \forall n \neq k \in\{1 \ldots 5\}$. In the event of a tie, the leader selects the method it voted for during the selection process. Once the leader settles on a capturing method, it needs to determine which agents it will allow to participate in the process. The next section discusses how suitable agents are chosen for a particular method through an auction.

\subsection{Agent Selection Through Auction}

As mentioned in Pryor and Norris (1998), not all dolphins are involved in the capturing phase and it is possible that the only the hungriest dolphins are the ones that participate. To model this, we need to develop an agent selection mechanism once the appropriate method is chosen. Assume that after the voting process, method $k$ is chosen to capture the prey. As mentioned in the previous sub-section, method $k$ requires $\left[N_{k}, N_{k+1}\right.$ ) agents and to select the appropriate number of agents, we design a simple auction (for more details on auctions, see Osbourne (2002)) where the leader agent is the auctioneer and the prize of the auction is the opportunity to participate in the prey-capturing process.

The bid of each agent for the prize is determined by its "hunger." If $b_{i}$ represents the bid placed by agent $i$, then we have $b_{i}=h_{i}(t)$, where $h_{i}(t) \in \mathbb{R}$ is the hunger coefficient of agent $i$. The hunger coefficient of the agent evolves as

$$
\dot{h}_{i}(t)= \begin{cases}h_{\max }-h_{i}(t) & \text { if } i \text { not eating fish at time } t \\ 0 & \text { otherwise }\end{cases}
$$

where $h_{\max }>0$. A simple first-order ordinary differential equation is chosen to keep an already complicated model from becoming more complex. Every time instance that agent $i$ does not capture fish, its hunger coefficient increases; it resets to 0 when it captures fish.
We let $v_{i}$ be the valuation of the prize by agent $i$, which represents how much the prize is worth to agent $i$. We model the valuation as a function that increases with the energy an agent has to exhaust to capture the prey. In our model, the energy exhausted is considered to be a function of the distance the agent needs to travel to start the capturing process. We use a simple formulation for valuation and let $v_{i}=\alpha\left\|x_{i}-\rho\right\|$, where $x_{i}$ is the position of agent $i, \rho$ is the centroid of the school of fish, and $\alpha$ is a scalar.

The utility (or payoff) for agent $i$ is given by $u_{i}=v_{i}-b_{i}$. Since the idea is restrict all the agents from joining the hunting group, we want the agents to first determine whether the hunt is even worth their participation. If the utility is positive, the agents will let the leader know that they are available for the hunt and our agents accomplish this through the function avail $: \mathcal{H} \rightarrow\{0,1\}$, defined as follows:

$$
\operatorname{avail}(i)=\left\{\begin{array}{lll}
1 & \text { if } & u_{i}>0 \\
0 & \text { if } & u_{i} \leq 0
\end{array}\right.
$$

The set of all available agents given by $\mathcal{A}$, where $\mathcal{A}=$ $\{i \mid$ avail $(i)=1 \forall i \in \mathcal{H}\}$ represent the agent that are eager to participate in the capture phase of foraging since they will receive a positive payoff. There are three cases that arise based on the number of agents in the set $\mathcal{A}$ and the leader selects agents as follows:

Case 1. $|\mathcal{A}|>N_{k+1}$. In this case, the leader selects the agents from the set $\mathcal{A}$ with the $N_{k+1}$ highest bids.

Case 2. $|\mathcal{A}| \in\left[N_{k}, N_{k+1}\right)$. The leader will select all the agents from the set $\mathcal{A}$.

Case 3. $|\mathcal{A}|<N_{k}$. In this case, the leader will select all the agents from the set $\mathcal{A}$ and to fill $N_{k}$ positions it will force $N_{k}-|\mathcal{A}|$ agents with the next highest bids (even if their utilities are negative) to join in the capturing process.

The dominance of agents will be used as a tie-breaker, i.e., for the same bid, the agent with a higher dominance will be selected over the agent with the lower dominance. Notice that in the third case, agents in the set $\mathcal{A}$ benefit from foraging with the herd, which is often the case with social foragers (Giraldeau and Caraco (2000)).

We now have a formulation to reproduce each of the three phases of foraging in a multi-agent system and in the next section, we present a hybrid automaton model of the the entire foraging process.

\subsection{Foraging Model as a Hybrid Automaton}

A hybrid automaton is used to model a dynamic system with both continuous and discrete variables (for example, see Henzinger (1996)). Since agents might enter or leave the different phases of the foraging process, the system dynamics will undergo discrete transitions. Hence, we model the foraging process as a hybrid automation, where the continuous dynamics unfold within the discrete states as seen in Figure 6. The transition from the state fuse to any of the states in the search phase depends primarily on the distribution of predators. The transition to detect phase depends on the distribution of prey and the transition to the capture phase depends on the number of agents available. Also, at anytime during foraging, if the threat level assessed by any group leader is greater than $r_{\max }$, then the agents will abandon the foraging process and return to the state fuse. 


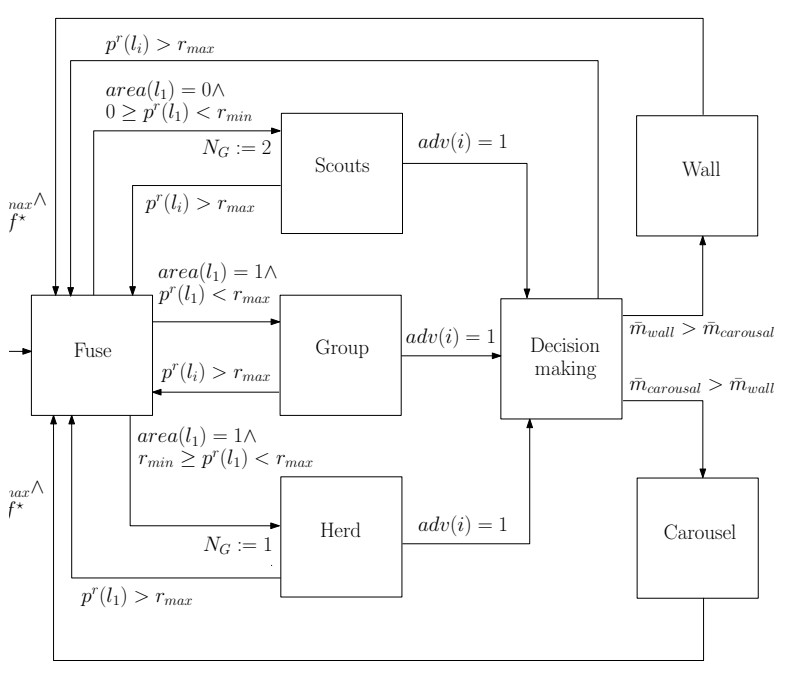

Fig. 6. Hybrid automaton model of the entire foraging process.

\section{PREDATOR AND PREY DYNAMICS}

In this paper, both predator and prey are assumed to have unicycle dynamics. Speed of the fish $S_{f_{i}}$ is assumed to be constant, while we let predators adjust their speed $S_{p_{i}}$ based on the mode they are executing. Hence, dynamics of the agents are of the form

$$
\left\{\begin{array}{l}
\dot{x}_{i}=S_{i} \cos \left(\theta_{i}\right) \\
\dot{y}_{i}=S_{i} \sin \left(\theta_{i}\right) \\
\dot{\theta}_{i}=\omega_{i}
\end{array}\right.
$$

where $i \in\left\{f_{1}, \cdots, f_{n}, p_{1}, \cdots, p_{N}\right\}$. Indices $f_{j}$ and $p_{j}$ represent fishes and predators respectively. A fish changes its heading only when it is closer than $\delta$ to other fishes, or when it is inside the influence arc of one or more dolphins.

Definition 1. The influence arc of a dolphin is an arc of radius $r$ and half angle $\alpha$, with its tip at the current position of the predator and its center line along the velocity vector of the predator.

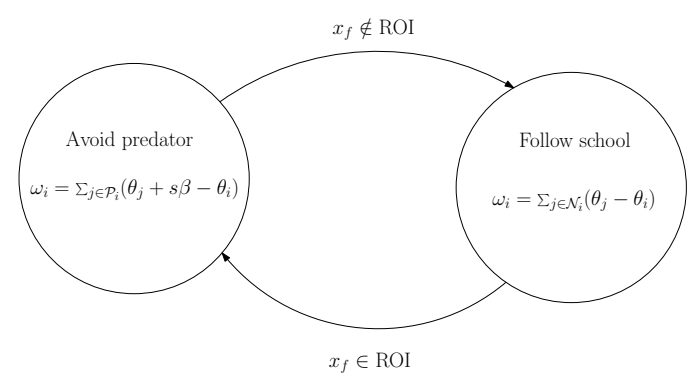

Fig. 7. Hybrid automaton governing fish dynamics.

When a fish is inside the influence arc of a predator, it tries to escape from the predator by turning towards the perpendicular vector to the velocity of the dolphin. We model this behavior as the fish running consensus protocol on the heading with the heading of the dolphins influencing it plus an angle $\beta \in\left(0, \frac{\pi}{2}\right]$. Our simulations show that fixing $\beta=\pi / 4$ generate results that are comparable to empirical data. When the fish is not inside the influence arc of any of the predators, it runs consensus protocol with neighboring fish within $\delta$ distance from it. The latter term models the schooling of the fish. This behavior of the prey constitutes a hybrid automaton depicted in figure 7 and is driven by

$$
\begin{array}{r}
\omega_{i}=\left\{\begin{array}{rr}
\sum_{j \in \mathcal{P}_{i}}\left(\theta_{j}+s \beta-\theta_{i}\right) & \text { if } \mathcal{P}_{i} \neq \emptyset, \\
\sum_{j \in \mathcal{F}_{i}}\left(\theta_{j}-\theta_{i}\right) & \text { otherwise, },
\end{array}\right. \\
\forall i \in\left\{f_{1}, \cdots, f_{n}\right\} .
\end{array}
$$

Parameter $s \in\{-1,+1\}$ indicates in what half of the influence arc the fish is located. Sets $\mathcal{P}_{i}$ and $\mathcal{F}_{i}$ are, respectively, the set of dolphins that fish $i$ is in their influence arc and set of other fish that their distance to fish $i$ is closer than $\delta$.

Motivated by the horizontal carousel formation of the bottlenose dolphins, we uniformly place them on a circle encompassing the school of fish. They swim counter clockwise with a constant speed, while spiraling toward the center of the circle by reducing their turn radius linearly, i.e.,

$$
\begin{aligned}
\dot{R}_{i} & = \begin{cases}-\alpha & \text { if } R_{i}>R_{\text {min }}, \\
0 & \text { otherwise, }\end{cases} \\
w_{i} & =S_{i} / R_{i} \quad \forall i \in\left\{p_{1}, \cdots, p_{N}\right\} .
\end{aligned}
$$

In three variation of wall method (fish in front, dolphin group as wall, two frontal attack), dolphins form a column and herd the fish toward the shore or another column of dolphins. Heading of the dolphins in these modes is constant, i.e. $w_{i}=0$ and they slow down as they get close to the shore or the other column of dolphins.

In the two column variation of wall method a column of dolphin attacking the fish surrounds the school from both sides and then move towards the shore. In our model, a column of dolphins moves towards the school of fish, when they get to a critical distance of the fish centroid, they alternate to go to the right and left side of the school of fish (odds to the right, evens to the left). Then, they adjust their headings to go straight again. They time this action so that their ROI overlaps and covers all the region in between the two column of dolphins. Heading dynamics of the prey in this mode is expressed as

$$
\begin{aligned}
& w_{p_{1}}(t)= \begin{cases}S_{p} / R & \text { if } t<t_{c_{1}}, \\
-S_{p} / R & \text { if } t_{c_{1}} \leq t<t_{c_{2}}, \\
0 & \text { otherwise, }\end{cases} \\
& w_{p_{i}}(t)=w_{p_{1}}(t+(i-1) \eta) .
\end{aligned}
$$

where $R$ is their turn radius and $\eta$ is the delay in the execution of the command. Parameters $t_{c_{1}}$ and $t_{c_{2}}$ characterize the time it takes to surround the school of fish and the time it takes to go back to the straight swim and are determind by the speed and turn radius of the dolphins to ensure there is no gap in the coverage of ROIs between the two formed columns of the dolphin.
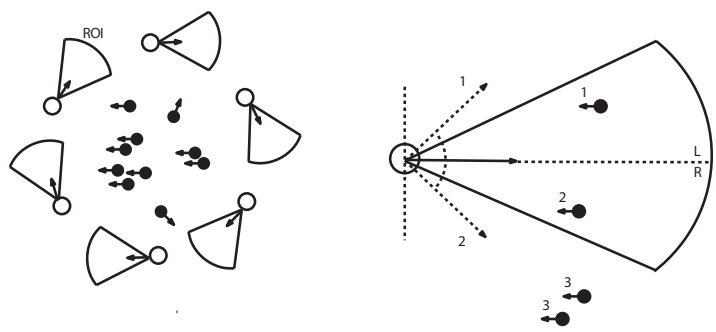

Fig. 8. Left: The region of influence (ROI) of the dolphins (white) are shown. Right: Fish (shown in black) inside the ROI run consensus with the projected dolphin heading, depending on which side of the ROI they lie (fish heading 1 is running consensus with dolphin heading 1 ). Fish outside the ROI run consensus only with their neighbors in the school (fish heading 3 is running consensus with fish heading 3 ). 

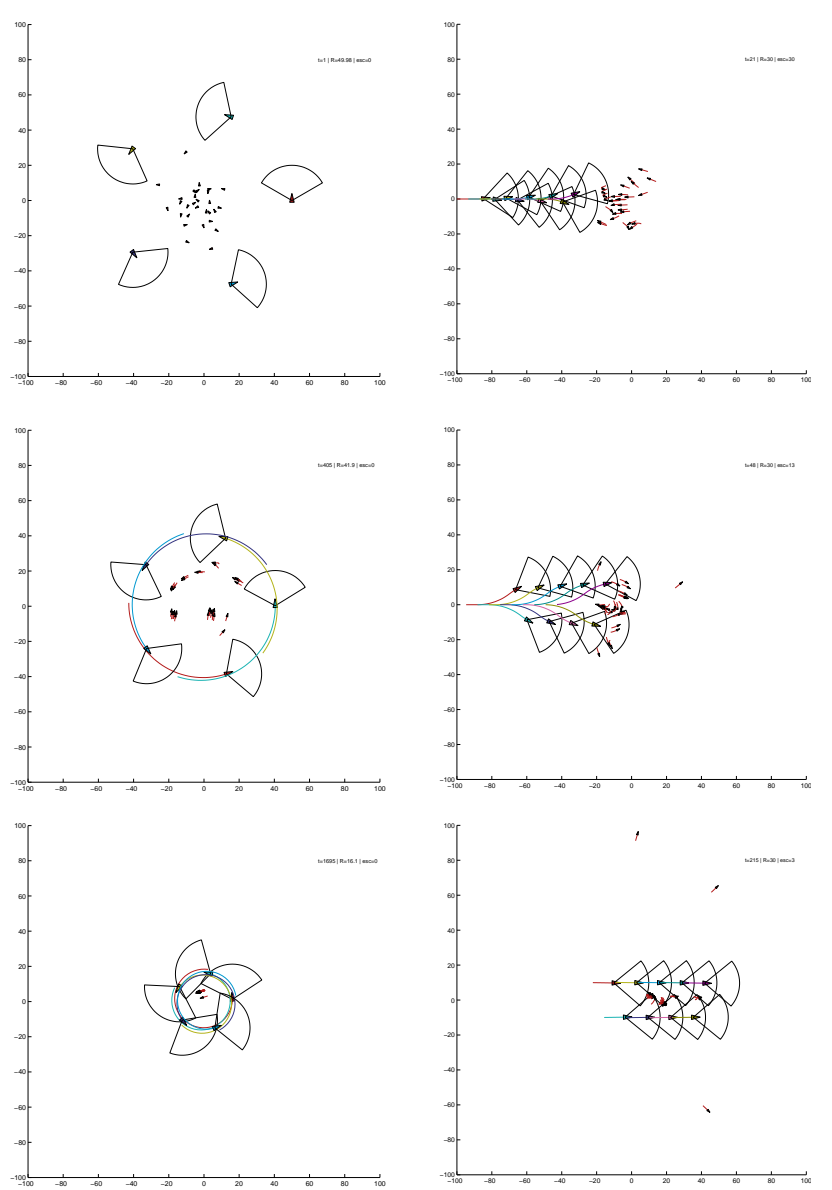

Fig. 9. School of fish entrapped in the bottlenose dolphins horizontal carousel (left) and two column wall method (right)

\section{SIMULATIONS}

We randomly initialize a group of $n=30$ fish inside a disk or radius 30 . In the horizontal carousel mode, dolphins start the encirclement on a circle of radius 50 around the fish. We assume influence arc of a dolphin has radius $r=10$ and half angle of $\alpha=.3 \pi$. Figure 9 (left) depicts the evolution of the system. This figure shows how fish get entrapped inside the dolphins carousel. The same model provide accurate results for dolphins wall formation and front formation, two other foraging techniques used by the bottlenose dolphins. Figure 9(right) shows how a column of dolphin split to trap the fish inside them and herd them towards the shore in the "two column" variation of the wall method. Figure 10 illustrates the "dolphin as wall" method in action where a group of dolphin push the school of fish towards another column of dolphin to feed on them.
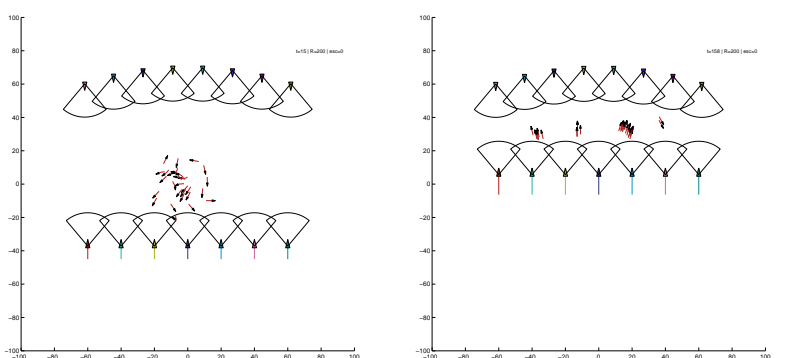

Fig. 10. Dolphins feed on the fish bounced back from the shore or another column of dolphin acting as wall

\section{CONCLUDING REMARKS}

Bottlenose dolphins search for fish by either sending scouts, in groups, or altogether in a herd and they either use the wall method or the horizontal method to capture their prey. We produce a hybrid automaton model of the entire foraging process in the context of heterogeneous multi-agent herding. Agents are modeled as first-order networks and their interactions are defined through a proximity graph. Finally, simulations are provided to show that our model is rich enough to mimic this complex biological phenomenon.

\section{REFERENCES}

J. Cortes, S. Martinez, and F. Bullo. Robust Rendezvous for Mobile Autonomous Agents via Proximity Graphs in Arbitrary Dimensions. IEEE Transactions on Automatic Control, 51(8):1289-1298, 2006.

J. Cousteau and P. Diol. Dolphins. Doubleday \& Company, Garden City, NY, 1975.

I.D. Couzin and N.R. Franks. Self-organized lane formation and optimized traffic flow in army ants. Proceedings of the Royal Society of London, Series B 270, pages 139-146, 2003.

I.D. Couzin. Behavioral ecology: social organization in fissionfusion societies. Current Biology, 16:169-171, 2006.

G. Ferrari-Trecate, M. Egerstedt, A. Buffa and M. Ji. Laplacian Sheep: A Hybrid, Stop-Go Policy for Leader-Based Containment Control. Hybrid Systems: Computation and Control, Springer-Verlag, pp. 212-226, March 2006.

L-A. Giraldeau and T. Caraco. Social foraging theory. Princeton University Press, Princeton, NJ, 2000.

M. Haque and M. Egerstedt. Decentralized Formation Selection Mechanisms Inspired by Foraging Bottlenose Dolphins. Mathematical Theory of Networks and Systems, Blacksburg, VA, July 2008.

M. R. Heithaus and L. M. Dill. Food Availability and Tiger Shark Predation Risk Influence Bottlenose Dolphin Habitat Use. Ecology, 83(2):480-491, 2002.

T.A. Henzinger. The theory of Hybrid Automata. Proceedings of the 11th Annual Symposium on Logic in Computer Science (LICS), IEEE Computer Society Press, pages 278-292, 1996.

D.J. Hoare, I.D. Couzin, J-G. Godin, and J. Krause. Contextdependent group size choice in fish. Animal Behaviour, 67: 155-164, 2004.

T. H. Labella, M. Dorigo, and J. Deneubourg Self-organised task allocation in a group of robots. Distributed Autonomous Robotic Systems, Toulouse, France, June 2004.

J. Mann, R. Connor, P. Tyack, and H. Whitehead. Cetacean Societies. The University of Chicago Press, Chicago, 2000.

M. J. Osbourne. An introduction to game theory. Oxford University Press, New York, NY, 2004.

E. H. Østergaard, G. S. Sukhatme, and M. J. Matarić Emergent Bucket Brigading. Proceedings of the fifth international conference on Autonomous agents, Montreal, Canada, 2001.

K. Pryor and K. Norris. Dolphin Societies. University of California Press, Berkely, CA, 1998.

R. Schusterman, J. Thomas, and F. Wood. Dolphin Cognition and Behavior: A Comparative Approach. Lawrence Erlbaum associates, Publishers, Hillsdale, NJ, 1986.

Y. Uny Cao, A. S. Fukunaga, and A. B. Kahng. Cooperative Mobile Robotics: Antecedents and Directions. Autonomous Robots, 4(1):7-27, 1997.

C. S. Wardle and J. J. Videler. How do fish break the speed limit? Nature, 284:445-447, 1980. 